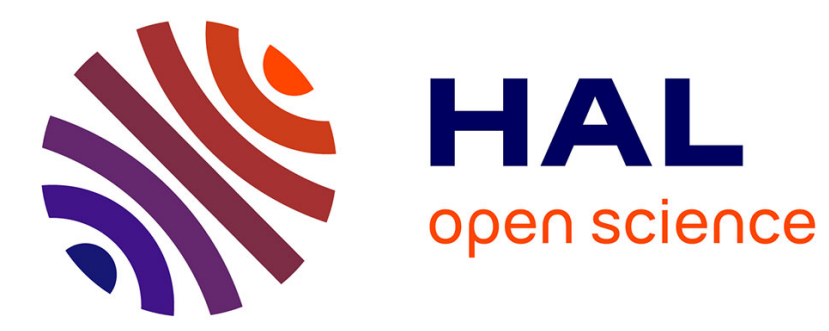

\title{
Quand la passion autorise la transitivation d'un circonstanciel de lieu
}

Anne Condamines

\section{To cite this version:}

Anne Condamines. Quand la passion autorise la transitivation d'un circonstanciel de lieu. Journal of French Language Studies, 2013, 23 (3), pp.335-356. halshs-00924832

\section{HAL Id: halshs-00924832 \\ https://shs.hal.science/halshs-00924832}

Submitted on 7 Jan 2014

HAL is a multi-disciplinary open access archive for the deposit and dissemination of scientific research documents, whether they are published or not. The documents may come from teaching and research institutions in France or abroad, or from public or private research centers.
L'archive ouverte pluridisciplinaire HAL, est destinée au dépôt et à la diffusion de documents scientifiques de niveau recherche, publiés ou non, émanant des établissements d'enseignement et de recherche français ou étrangers, des laboratoires publics ou privés. 


\section{Quand la passion autorise la transitivation d'un circonstanciel de lieu Anne Condamines}

Résumé

L'article s'intéresse aux cas d'énoncés dans lesquels un complément circonstanciel (de lieu) se trouve mis en position d'objet. L'hypothèse qui est étudiée est que ces énoncés, qui paraissent très inattendus, sont possibles dans des situations de communication bien particulières, celles qui, associées à des domaines spécialisés, se déroulent dans un contexte de connivence très fort. Le locuteur qui transitivisele complément fait alors le choix de mettre en exergue le lieu dans lequel se déroule l'action plutôt que l'objet. L'article décrit ainsi le cas de la pêche. A partir des occurrences attestées sur la toile concernant les relations entre pêcher et rivière, il s'avère d'une part que la structure pêcher une rivière, en principe a-normale, est relativement fréquente et, d'autre part, qu'elle apparaît majoritairement dans des sites personnalisés (de type blogs ou forums). Ces éléments, associés à d'autres comme l'analyse du fonctionnement de l'adjectif pêchable, confirme l'hypothèse et ouvre de nombreuses perspectives, vers l'analyse discursive, vers la sémantique et vers la terminologie. 


\section{Quand la passion autorise la transitivation d'un circonstanciel de lieu}

\section{1- Introduction}

Le phénomène de la transitivité a fait l'objet de nombreux travaux tant du point de vue syntaxique que du point de vue sémantique. Celui de la transitivation a été nettement moins exploré. Il concerne les cas où un complément circonstanciel, «normalement » construit à l'aide d'une préposition se trouve directement mis en relation avec le verbe.Nous explorons l'hypothèseque la transitivation d'un circonstanciel de lieu dans les domaines spécialisés est liée à une situation de communication particulière. En effet, nous pensons que, dans ces domaines spécialisés, le choix du locuteur de mettre en position d'objet un élément qui ne devrait pas s’y trouver «normalement » est à mettre en relation avec le lien primordial que le locuteur veut mettre en exergue entre le sujet (souvent lui-même) et l'élément qu'il place en position d'objet. Nous pensons que le locuteur ne s'autorise cette transgression que dans des situations de communications particulières, dans lesquelles il partage (ou veut faire partager) une passion, c'est-à-dire une situation qui demande une implication forte du sujet par rapport à un objet. Les verbes concernés relèvent d'une activité particulière et peuvent donc être considérés comme des termes mais la construction apparemment « déviante » dans laquelle ils apparaissent nécessite de faire intervenir une notion de genre textuel qui prenne en compte à la fois le domaine et l'idée d'une forte implication du locuteur, ce qui est assez inédit dans les études sur les discours spécialisés.

Afin d'examiner cette hypothèse, nous avons mis en place une étude qui analyse un cas original de domaine, celui de la pêche, qui, dans certaines circonstances, autorise la transitivation suivante : «pêcher det rivière » alors qu'on attendrait un circonstanciel de lieu : «pêcher dans det rivière ». Nous avons mené une recherche à partir d'attestations sur le web afin d'obtenir un grand nombre de données et de pouvoir tenir compte, pour l'analyse, de la nature des textes dans lesquels cette structure inattendue apparaît.La partie 2 présente la mise 
en place de l'étude dans le domaine de la pêche et la partie 3 détaille les résultats obtenus et les pistes de travail à venir.

2- Hypothèses et mise en place de l'étude

Cette partie s'intéresse d'une part,aux travaux sur la transitivité et la transitivation et, d'autre part, elle présente la mise en place de l'étude à partir d'analyses d'occurrences issues de l'internet

2-1 La transitivité et la transitivation

La question de la transitivité a fait l'objet de nombreux travaux, tant syntaxiques que sémantiques:" Grammaticalement, on définit comme «" transitifs" les verbes qui peuvent ou doivent être accompagnés d'un objet'. (Lazard, 1994: 248)

'Transitivity is "how events and processes are connected (or not connected) with subjects and object".'(Jørgensen et al., 2002: 83)

Certains auteurs évoquent même la nature universelle de ce phénomène :

'La transitivité sémantique peut être définie en termes universels. Elle implique " un agent contrôleur et effectueur et une situation cinématique qui affecte un patient qui ainsi change d'état".'. (Desclés, 1998 : 166).

La plupart des linguistes acceptent l'idée d'une gradation dans la transitivité (Lakoff, 1997), (Dowty, 1991), les énoncés se situant dans une plus ou moins grande proximité avec une «transitivité prototypique ». Dans leur célèbre article de 1980, Hopper et Thompson proposent dix «caractéristiques» sémantiques permettant d'évaluer la transitivité d'un énoncé : participants, kinesis, aspect, punctuality, volitionality, affirmation, mode, agency, affectedness of $\mathrm{O}$, individuation of $\mathrm{O}$. Selon le nombre de critères présents dans un énoncé, on peut décider s’il est plus ou moins transitif. 
Par ailleurs, de nombreux auteurs signalent qu'un verbe réputé intransitif peut être utilisé avec un complément direct

'Un autre verbe, réputé intransitif, comme courir admet lui aussi bon nombre d'objets :

[...] Courir le 100 mètres, [...] courir les haies, [.. ;] courir la finale, [.. ;] courir la fille, [...] courir le risque/la chance'. (Rousseau, 1998: 87).

D'autres auteurs remarquent que des verbes construits avec une préposition peuvent se trouver construits directement (penser à detX/penser detX, monter un cheval/monter sur uncheval) (François, 2003). (Larjavaara, 2008) étudie le lien entre ces variations et le genre textuel en concluant que 'On constate donc une différence d'usage, les textes non littéraires mais hautement normatifs profitant de la possibilité d'une construction transitive du verbe penser'.Dans sa thèse, cette même auteur parle de « relation dénudée » pour évoquer les cas où ' l'objet $[\ldots]$ dénote un référent auquel pourrait renvoyer un syntagme prépositionnel, la construction avec préposition étant plus conventionnelle '. (Larjavaara, 2000 : 138).

Dans le même ordre d'idée, (Serbat, 1994) note le cas de jouer dans le domaine du golf (mais ce serait probablement vrai pour tous les sports), qui peut se trouver construit directement avec un grand nombre de compléments directs correspondant à des éléments considérés comme majeurs pour la pratique de l'activité :

'L'exemple de ce français correct montre que la place syntaxique d'objet est accessible au nominal qui reflète une partie du signifié sémantique du verbe lui-même [...]. Du coup, l'énoncé met en relief, parmi tous les autres, celui des traits du verbe qui convient à son N1 : «jouer le Basque » estompe terrain, matériel etc. pour donner le premier rang à une image de compétition'. (Serbat, 1994: 58).

Comme il le détaille dans (Serbat,1998), au fond, ce qui se manifeste dans ce phénomène de transitivation, c'est l'importance que le locuteur accorde aux différents éléments en jeu dans 
l'activité.En effet, il est évident que l'élément qui se trouve en position d'objet acquiert un rôle de focalisation.

Nous voulons dans la suite de l'article montrer que, dans des domaines spécialisés, la possibilité de cette transitivation(et plus particulièrement de la transitivation d'un complément de lieu) est liée à l'implication «affective» du locuteur (et celle qu'il suppose de son interlocuteur). Le résultat de ce type de transitivation ne correspond pas à la structure normée et le locuteur ne peut se l'autoriser que dans des situations adaptées, situations dans lesquelles il se sent autorisé à « dévier » de la norme.Deulofeu a repéré aussi ces formes de transgression dans une situation où l'implication émotionnelle est forte :

'Dans les passages où l'émotion l'emporte, les reporteurs ne se sentent pas obligés de se conformer à des modèles syntaxiques normatifs'. (Deulofeu, 2000 : 275).

C'est certainement un processus similaire qui se met en place dans les phénomènes de transitivation.

\section{2-2L'internet comme pseudo-corpus}

Afin de diversifier l'origine des occurrences à observer, nous avons pris le parti d'utiliser l'internet comme source d'exemples attestés en faisant des recherches grâce au moteur de recherche Google.

L'objectif est de tenir compte à la fois du nombre d'occurrences et du type des sites dans lesquels elles sont repérées. En ce qui concerne la dimension quantitative, il est clair qu'il ne faut prendre les données attestées que comme une tendance; nous savons en effet que les propositions des moteurs de recherche présentent des difficultés majeures et que le web ne peut pas être considéré comme un corpus, mais tout au plus comme un pseudo-corpus :

'Le pseudo-corpus Web ne garantit pas qu'une fréquence élevée pour une forme corresponde à un usage répandu et ce, à cause de phénomènes bien spécifiques (documents dupliqués, 
citations, migrations de sites et indexations séparées dans le temps, absence d'identification de l'auteur etc...)' (Hathout et al., 2003: 58).

Toutefois, d'une part, lorsque ces tendances sont marquées, elles peuvent être considérées comme significatives, d'autre part, ce qui nous intéresse, ce sont surtout les liens entre la présence de telle ou telle occurrence et le type de site dans lequel elle apparaît.

Rappelons que la nature des recherches linguistiques possibles sur internet est contrainte puisqu'elles ne peuvent se faire que sur des formes exactes. Les formes de variation,qu'elle concernele temps ou les personnes pour les verbes, le nombre ou la nature des déterminants pour les syntagmes nominauxdoivent donc être recherchées systématiquement.

Enfin, il est évident que le web n'est pas représentatif de l'ensemble des situations de communication et donc de l'ensemble des genres textuels.Dans notre perspective, il eût mieux valu disposer d'un corpus avec des attestations d'oral, qui sont plus propices à instaurer un contexte émotionnel particulier. Mais, d'une part, nous ne disposons pas d'un tel corpus et, d'autre part, nous avons fait l'hypothèse que certains genres de l'internet favorisent l'implication personnelle des locuteurs d'autant qu'ils se savent couverts, s'ils le désirent, par l'anonymat.

Un certain nombre de travaux, basés sur des traits linguistique repérables automatiquement (qui s'inspirent beaucoup de la méthode de caractérisation «à la Biber » (Biber, 1988)) ont permis d'établir des listes de genres présents sur le web. Citons par exemple la liste établie par Karlgren et son équipe (Karlgren et Cutting, 1994) en prenant en compte à la fois des traits linguistiques et les « impressions des internautes », liste reprise dans (Beauvisage, 2001):

- pages personnelles (« informal, private : personal home pages»);

- sites commerciaux («public, commercial : home pages for the general public»);

- pages interactives (« interactive pages : pages with feed-back : searchable indexes, customer dialogue») ; 
- matériel journalistique («journalisticmaterials : press : news, editorials, reviews, ezines ») ;

- rapports (« reports : scientific, legal and public materials ; formaltext »);

- autres textes (« othertexts »);

- FAQ («FAQs »);

- pages de liens (« link collections »);

- autres tableaux et listes (« other listings and tables »);

- forums de discussion (« dicussions »);

- messages d'erreur («error messages »).

Nous verrons que notreclassification des sites, que nous avons retenue sur des bases uniquement intuitivespour notre étude, recoupe en grande partie cette liste.

2-3Genre textuel, variation terminologique, jargons

L'approche «classique » de la terminologie prend très peu en compte la variation. En effet, selon la théorie de Wüster (1981), la vision prescriptive de la terminologie permet de conserver la transparence de la communication. Toutefois, depuis une vingtaine d'années, en lien avec le développement de la linguistique de corpus et des outils de traitement automatique de la langue, les analyses du fonctionnement des termes se font de plus en plus souvent à partir d'usages attestés. La prise en compte de la variation est alors inévitable, qu'elle soit liée à l'évolution dans le temps, à l'interdisciplinarité, à la variation des registres de langue...

Une des variations en terminologie qui commence à être admise est celle qui est liée au genre textuel (peut-être plutôt au registre) (Rogers, 2000). Des travaux ont été menés sur la variation des termes eux-mêmes. On sait ainsi qu'en fonction du statut des locuteurs et des interlocuteurs et de leur niveau d'expertise, des variations existent quant au choix (plus ou moins conscients) des dénominations. 
Plus récemment, les études ont porté sur la variation des «marqueurs de relations conceptuelles » en fonction du genre textuel. La question est plus complexe que la seule variation dénominative. En effet, elle concerne des structures lexico-syntaxiques pour lesquelles la possibilité d'une variation n'avait même pas été envisagée (Condamines, 2002), (Marshman et al., 2008). Nous avons ainsi repéré de ce point de vue un fonctionnement très particulier du marquage de la méronymie dans les petites annonces immobilières (Condamines, 2009).

Quelle que soit l'étude (dénominations ou marqueurs), la notion de genre qui est adoptée est celle qui correspond peu ou prou à la définition proposée par (Bahtia et al., 1993: 13) 'itis a recognizable communicative eventcharacterized by a set of communicative purpose(s) identified and mutuallyunderstood by the members of the professional or academiccommunity in whichitregularlyoccurs.'

Si, pour la langue générale, certaines études sur le genre ont porté sur l'implication émotionnelle du locuteur (voir par exemple les travaux sur l'autobiographie (Baudouin, 2004)), aucune étude, à notre connaissance, n'a pris en compte cette dimension pour expliquer des variations dans des domaines spécialisés. En effet, ces domaines, souvent assimilés à des domaines techniques ou scientifiques sont censés relever d'une norme langagière en lien avec la stabilité des connaissances qui y sont à l'œuvre. Pour le dire autrement, il s'agit de domaines supposés impersonnels, sans présence «impliquée » des locuteurs, qui s'effacent derrière la connaissance partagée avec les autres experts du domaine. Ainsi, les manuels techniques (assez représentatifs des genres étudiés dans les domaines spécialisés) sont-ils réputés pour ne jamais utiliser les pronoms de première et deuxième personnes.Les seuls travaux (rares) qui s'intéressent aux phénomènes (seulement lexicaux) en situation de connivence sont ceux qui concernent les «jargons »(Depecker, 1994), mais la plupart du temps, le découpage terme/jargon ne se fonde que sur la dimension 
« valorisé »(parce que technique ou scientifique) vs «dévalorisé » (parce qu'utilisé dans des contextes prétendument moins nobles). Nous rejetons cette dichotomie parce que nous pensons que la connivence existe même dans des contextes scientifiques ou techniques.

3- Etudesdans le domaine de la pêche

Afin d'évaluer notre hypothèse, nous avons choisi de travailler sur le domaine de la pêche. Il s'agit bien d'un domaine spécialisé, même s'il relève souvent plutôt d'un loisir, qui a ses experts et ses propres usages langagiers.

Pour ce qui concerne cette activité, on pourrait dire que la structure « cognitivo-linguistique » attendue, considérée donc comme standard est la suivante :

$$
\text { [X pécher Y dans } \mathrm{Z} \text { avec } \mathrm{W}]
$$

C'est en effet celle que l'on trouve dans les exemples suivants :

1. je pêche la truite dans les lacs du val d'Aran(dans lequel il manque W).

2. on peut pêcher la carpe avec un flotteur dans les étangs ou lacs qui sont vraiment vaseux.

On peut aussi admettre comme normés des cas où dans est remplacé par en :

3. J'affectionne surtout de pêcher en rivière.

Nous avons aussi trouvé des cas qui semblent moins normés dans lesquels sur remplace dans.

4. Je vais aller pêcher sur la rivière Yamaska à Granby samedi s'il ne pleut pas.

Dans quelques cas enfin, avec la préposition sur, le déterminant disparaît :

5. Pour avoir essayé une thébaut et une newfly parallèle toutes les deux en condition de pêcher sur rivière [...]

Dans ces réalisations, on trouve :

$\mathrm{X}$ : humain (ou en tout cas, animé), $\mathrm{Y}$ : poisson ou crustacé, $\mathrm{Z}$ : cours d'eau ou étendue d'eau ou espace contenant ce type d'espace aqueux, W : méthode, instrument de pêche ou appât.

D’un point de vue sémantique, des glissements sont possibles : 
$\mathrm{X}$ peut être un collectif

6. L'Algérie pêche presque 230000 tonnes par an, selon le ministère de la Pêche et des Ressources halieutiques.

Y peut être un objet que l'on trouve dans W (autre qu'un poisson),

7. Eh oui, on peut pêcher des objets rares, la chance d'en pêcher augmente avec le niveau de votre canne.

Enfin, l'énoncé peut être utilisé de manière métaphorique, comme dans l'exemple 8 . Mais ces cas sont très rares et nous ne les avons pas pris en compte dans la suite de notre étude.

8. Certains pourraient être tentés d'aller pêcher sur internet le contenu d'un paragraphe.

En revanche, il semble difficile de trouver en W autre chose qu'une méthode, un instrument ou un appât.

Ce à quoi nous allons nous intéresser par la suite concerne les modifications de constructions syntaxiques qu'elles concernentla « construction avec préposition » de Y ou(ce sera le focus de notre article) la transitivation de $\mathrm{Z}$ comme dans :

9. Un droit de pêche journalier est requis pour pêcher la rivière, que vous pouvez vous procurer entre autre au Métropole.

3-1 Construction transitive directe vs indirecte : pêcher (à) det $\mathrm{N}$ de poisson. pêche de/à det N de poisson

Cette partie ne concerne pas le phénomène de transistivation du complément de lieu mais l'alternance de la construction directe vs indirecte de l'objet «normalement » concerné par la pêche : le poisson. Il s'agit de casoù, dans la structure contenant un verbe, la construction attendue est une construction directe alors qu'une préposition apparaît ou encore, dans la structure contenant la nominalisation déverbale, alors que c'est la préposition de qui devrait être utilisée, c'est à qui apparaît. Notre objectif ici est de montrer que la prise en compte 
d'usages attestés sur l'internet amène à revoir la notion de construction normée vs nonnormée, hypothèse qui sous-tend notre étude sur la transitivation des criconstanciels de lieu.

\section{3-1-1Structure contenant la nominalisation déverbalepêche}

Notre première recherche a porté sur la forme nominalisée du verbe dont on s'attendrait à ce qu'elle suive le schéma suivant :

\section{[(X réalise det) pêche de $\mathrm{Y}$ dans $\mathrm{Z}$ avec $\mathrm{W}]$}

Structure qui est présente dans 10 et 11.

10. pêche de la truite dans les lacs de montagne

11. quelques conseils pour la pêche de la truite avec des leurres

Nous avons tout d'abord recherché les structures [pêche du/de la] afin de constituer une liste de poissonsou de crustacés (voir tableau 5 en annexe) qui apparaissent en position de Y. Puis, nous avons utilisé systématiquement cette liste pour rechercher les structures contenant à [pêche au/à la] clairement en concurrence avec la structure en de.

12. La pêche à la carpe est une passion, je vais essayer de vous la faire découvrir

13. Pêche au crabe en eaux troubles.

La recherche via google montre que l'on trouve plus souvent la construction avec à [pêche à det poisson/crustacé] que la construction avec de (tableau 1).

Notons que, dans cette même construction avec $\grave{a}$, on peut aussi trouver en position de $\mathrm{N}$ :

- Un lieu :

14. Parties de pêche au lac de Monteynard (38) sur des criques où l'accès n'est possible qu'en bateau

- Un type d'appât ou de méthode

15. La pêche aux appâts naturels, autrefois et toujours d'ailleurs, nommée pêche au toc[...]

16. La pêche àla mouche des gros salmonidés prend également toute sa dimension dans les Pyrénées espagnoles. 
Le tableau 6 de l'annexe recensela liste des noms placés dans la positionY de la structure [pêche à le/la Y]à condition que Y ne soit ni un poisson, ni un lieu.Nous faisons l'hypothèse que ces noms correspondent à un mode de pêche : appât ou méthode (hormis quelques cas d'expression d'une période comme dans pêche au mouillage).

Ce type de construction apparaît comme moins inattendu que les énoncés dans lesquels Y est un poisson ou un crustacé. En effet, il est connu, que, en tant que préposition «incolore » (Spang-Hanssen, 1973) la préposition à peut introduire un lieu ou un instrument (on pourrait ainsi trouvercuisson à la plancha, cuisson à la boulangerie mais pas *cuisson au pain de mie).

Finalement, tous les compléments possibles du verbe pêcher peuvent se trouver dans la position du complément introduit par à dans la structure avec nominalisation déverbale : poisson, étendue d'eau(ou lieu dans lequel il y a une étendue d'eau), méthode, soit Y, Z ou W de la structure canonique.

L'élément le plus étonnant reste la fréquence de la structure qui concerne la catégorie poisson ou crustacé dont on s'attendrait à ce qu'elle se construise avec la préposition de.

Premier résultat étonnant donc puisque c'est la forme en principe la moins normée syntaxiquement qui manifeste le plus souvent la relation entre pêche et poisson.

\section{3-1-2 Forme verbale}

Dans la forme verbale, la préposition à peut aussi introduire toutes les catégories de compléments : méthode ou appât, lieu ou poisson (ce dernier cas correspondant à un cas de construction transitive indirecte) :

- Méthode ou appât : Pêcher à pied, à la ligne, à l'anglaise.

- Lieu : Pêcher à St Côme d'Olt, à l'étang du Tuquet, à l'île Maurice.

- Poisson : Pêcher à la carpe, à la mouche, à la sardine.

17. Par mer agitée, il est possible de pêcher à la sardine sans amorcer au préalable 
18. c'est déjàgalère de pêcher à la moule par temps calme, alors, en surf casting [...].

Toutefois, si l'on regarde le nombre de noms relevant de la catégorie poisson/crustacé, sur les 7531 occurrences, on trouve $89 \%$ de constructions directes, c'est-à-dire d'occurrences où les poissons-crustacés sont à leur place attendue d'argument direct. Le fonctionnement s'avère donc différent de celui de la nominalisation qui, pour la catégorie des poissons/crustacés, semble préférer la construction la moins normée alors que le verbe préfère la construction attendue.

Pour cette première étude, nous n'avons pas pris en compte le lien entre la fréquence d'apparition de la construction «déviante» (avec construction de la catégorie poisson/crustacé par la préposition à) et le genre textuel. Il semblerait en effet que le genre textuel soit beaucoup moins discriminant pour cette construction indirecteque pour celle que nous allons présenter dans le paragraphe suivant. Cette forme de construction indirecteest d'ailleurs «admise » dans le Trésor de la Langue Française.

3-2 Phénomène de transitivation : [Pêcher det rivière]

La structure que nous allons étudierplus en détail dans ce paragraphe correspond à la transitivationdu circonstanciel de lieu correspondant à l'étendue d'eau (courante ou pas) dans la structure canonique de la pêche. Plus précisément, nous nous sommes intéressée aux exemples :

[Pêcher det rivière] comme :

19. Difficile de pêcher une rivière que l'on connaît mal.

Pour des non-pêcheurs, cette structure avec construction directe semble vraiment improbable. Elle n'est d'ailleurs acceptée dans les dictionnaires que pour les pièces d'eau «fermées » avec un sens bien précis. Ainsi dans le TLF, on peut lire comme sens possible à l'entrée pêcher: Vider (un étang, une pièce d'eau) pour prendre les poissons:'Rien ne restait dans les campagnes, déjà ruinées par l'impôt (...); les viviers étaient pêchés'. 
Cette définition ne concerne donc que les étendues d'eau fermées qu'il s'agit de vider entièrement de leurs poissons.

Nous allons voir que cette structure est pourtant tout à fait présente sur l'internet, avec un autre sens.

Afin d'étudier de manière comparative les usages de [pêcher dans det rivière] et [pêcher det rivière], nous avons utilisé le moteur de recherche Google pour rechercher toutes les occurrences de :

- «pêcher det rivière» avec successivementdet $=$ la/les/une/des.

- «pêcher dansdet rivière » avec successivement det $=$ la/les/une/des

- «pêcher en rivière »

- $\quad$ «pêcher sur (det) rivière » avec successivement det $=$ la/les/une/des.

Nous n'avons donc pas pu récupérer les cas où le verbe est conjugué (donc non-prise en compte des personnes ni des temps) ni les cas (nombreux) où c'est le nom d'un cours d'eau qui est utilisé (pêcher le Lézert/pêcher dans le Lézert), ni les cas où c'est un autre type d'étendue d'eau qui est concerné (comme un lac, une mer ou un fleuve), ni les cas où le déterminant n'est ni un défini, ni un indéfini (mais un démonstratif par exemple).Bien que nous ayons travaillé sur 1474 occurrences, il ne s'agit donc que d'un échantillon, que nous espérons représentatif, de toutes les structures possibles impliquant le verbe pêcher et le nom rivière.

Pour analyser les liens entre genre et transitivation, nous avons voulu voir comment deux caractéristiques de la situation interviennent dans l'apparition de ces formes :

- Le domaine : pêche vs non-pêche.

- L'implication du locuteur : présence « affective »du locuteur.

En regardant le type de sites dans lesquels apparaissent les occurrences, nous avons repéré deux catégories: 
- d'un côté les sites personnalisés de type blog, pages personnelles, forums et récits ;

- d'un autre côté les sites non-personnalisés que nous avons répartis en presse, livres, agences de voyages, textes légaux, sites de traduction, dictionnaires, divers (publicités, jeux vidéo (l'activité de la pêche, pas forcément des poissons semble faire l'objet de plusieurs jeux vidéo !)).

Dans la plupart des cas, l'adresse même du site permet de repérer la nature du site (blog.mouche-fr, pechemaniac.com/forums/, pacvoyages.fr, pecheaveyron,...) ou le «titre » du site (« Directive sur l'exercice de la pêche », « Le Monde de la Truite n 6 », « Chambres d'hôtes à Cudillero », «Tourisme dans le Morvan », «Je suis anglais, pouvez-vous corriger mon français ? », «Règlements passage et pêche », «liste des ouvrages par année depuis $1972 »$, «Livres de poche », ...). Dans les autres cas, nous avons ouvert la page pour évaluer la nature du site.

Il est clair que ce classement des sites peut être difficile : dans un survol rapide, il n'est pas évident,par exemple,de distinguer un récit d'une publicité. Des indices dans le texte permettent pourtant rapidement de savoir si on a affaire à un site personnalisé ou non (présence des premières et deuxièmes personnes, d'exclamatives, d'un vocabulaire appréciatif dans les sites personnalisés).

Dans le classement proposé par Karlgren et son équipe, 3 types de sites relevaient de cet aspect «personnalisé »: les pages personnelles, les pages interactives et les forums de discussions, ce qui correspond à peu près à notre classement.

Voici quelques exemples trouvés grâce à ces recherches ainsi que la catégorie de leur site de provenance.

20. Nous mettons à votre disposition un bateau pour pêcher dans la rivière en bas du jardin, une table de ping-pong, un jeu de badminton, des fléchettes.(location de gîte). 
21. Une canne plus courte offre souvent plus de précision quand il s'agit de pêcher sur des rivières pas très larges. (blog).

22. Il nous proposa de pêcher la rivière Matane, cette dernière ayant donné plus de satisfaction les jours précédents. (récit).

23. Conçu directement pour pêcher en rivière, ce plomb «in line » allie longue distance et stabilité dans le courant.(divers, publicité).

24. Toute personne de plus de 16 ans qui souhaite pêcher dans des rivières où il y a du saumon doit payer des droits de pêche nationaux. (légal).

Le tableau 2 présente les résultats obtenus pour chacun des genres. Les trois premières lignes correspondent à des genres «personnalisés », les autres non. Pour chacune des 4 structures possibles : pêcher dans det rivière, pêcher en rivière, pêcher sur (det) rivière, pêcher det rivière, nous avons globalisé les résultats avec toutes les valeurs possibles des déterminants (la/les/une/des).

Ces résultats appellent différentes remarques :

- La forme la plus normée apparaît dans moins de la moitié des occurrences.

- La forme [pêcher det rivière] en principe considérée comme « déviante » est tout à fait attestée dans les occurrences extraites du web $(17,7 \%$ du total des occurrences).

- Les forums utilisent plus souvent la forme avec construction directe que la forme la plus normée (avec introduction de detrivière par dans).

- Les sites de traduction n'utilisent jamais la forme avec construction directe. Petite anecdote à ce sujet, un dictionnaire en ligne propose pour traduction de to fisha river : pêcher dans une rivière! Il semblerait que certains traducteurs aient tendance à normaliser ! 
- Les sites légaux n'utilisent qu'une fois la forme avec transitivation de rivière. Cet élément n'est pas étonnant dans la mesure où on s'attend à ce que les textes légaux soient aussi normalisés du point de vue de l'usage langagier.

- Les sites personnalisés, s'ils utilisent les quatre formes possibles, sont ceux qui utilisent le plus la construction directe (blog : 33,5\%, Forum : 30,5\%) ce qui constitue un début de confirmation de l'hypothèse d'un lien entre situation de communication personnalisée et transitivation du circonstanciel.

Toutefois, cette première étude n'a pris en compte que le rôle du genre dans la variation des types de construction entre pêcher et rivière mais pas celui du domaine. Ainsi, un forum parlant de la pêche n'est pas nécessairement un forum dédié à la pêche. Il en va de même pour un livre qui peut être soit un roman, soit un manuel de pêche. Dans un second temps, nous avons fait intervenir la dimension du domaine afin de distinguer les sites concernant la pêche et personnalisés (blogs, forums et récits) des autres. Par exemple, un forum sur le site du magasin de sport Décathlon (donc qui ne relève pas de la pêche, en tout cas pas prioritairement) fait apparaitre l'exemple suivant :

25. [La canne] n'est pas chère et je voulais savoir s'il y a un bon rapport qualité-prix pour pêcher en rivière.

Voici un autre exemple, tiré d'un roman en grande partie lié à la pêche (L'enfant et la rivière, d'Henri Bosco (disponible sur le web)) mais qui n'est ni un blog, ni un forum :

\section{Qu'il aimerait lui aussi pêcher sur la rivière!}

Le tableau 3 rend compte des résultats obtenus. Le premier chiffre concerne le nombre d'occurrences correspondant à la structure dans les sites personnalisés du domaine de la pêche ; le deuxième correspond au nombre total d'occurrences de cette structure trouvées sur le web. 
Il apparaît clairement que la construction directe apparaît très majoritairement (à plus de 75\%) dans les sites qui relèvent du domaine de la pêche et qui sont personnalisés. Le tableau 4 se focalise sur la répartition des quatre structures dans les sites personnalisés relevant de la pêche. On voit que la structure dominante est bien celle qui contient la construction directe mais les autres structures sont aussi possibles.

3-3Pêchable, un indice de transitivité

Traditionnellement, on associe l'existence d'un adjectif en -able avec le fait que cet adjectif renvoie, dans le cas d'un verbe transitif, au complément direct du verbe (on peut accepter cette réponse/cette réponse est acceptable) (Dubois, 1962). Toutefois, différents travaux (Leeman, 1992), (Fradin, 2003) ont montré que ce n'était pas toujours l'objet direct qui se trouvait ainsi adjectivé :

'Les linguistes et les lexicographes mentionnent peu souvent le fait que certains adjectifs able sélectionnent comme nom recteur un nom correspondant au complément prépositionnel du verbe dont ils dérivent'. (Hathout et al., $2003: 63$ ).

Nous avons voulu savoir ce qu'il en était pour pêchable. Premier constat, également signalé par Hathout et al. qui ont étudié cet adjectif : pêchable n'est pas recensé dans le TLF.

Or, nous avons trouvé 583 occurrences de cet adjectif sur l'internet :

- 458 associés à un cours ou une nappe d'eau (plus de $78 \%$ );

- 125 associés à un poisson ou un crustacé.

D’un point de vue syntaxique, on peut faire deux hypothèses : soit, le nom concerné par l'adjectif n'est pas toujours l'objet direct mais peut être 'tout élément intervenant dans le procès X' (Hathout et al.,ib. :49), soit c'est bien l'objet qui est retenu comme nom recteur de pêchable, ce qui suppose d'admettre une structure possible avec un élément de la catégorie rivièrecomme objet de pêcher. 
Le point essentiel pour nous est que le nom recteur de pêchablesoit très majoritairement le nom indiquant le lieu de la pêche (en position $\mathrm{Z}$ dans notre schéma global).

La notion de possibilité (généralement associée au suffixe -able) se décline en deux : d'une part, elle est reliée à la notion de légalité (c'est autorisé par la loi), d'autre part, elle est reliée à la notion de présence de conditions favorisant l'activité de la pêche. Le sens légal se trouve dans les sites légaux mais n'est pas impossible dans les sites de pêche. Ce qui est net, en revanche, c'est que ce qui intéresse majoritairement les pêcheurs, ce sont les conditions (météorologique, d'accessibilité, de nature du matériel, de période) qui font qu'ils peuvent (ou non) pêcher (dans) une rivière, ce qui va vers l'idée qu'au fond, ce qui intéresse les pêcheurs, c'est la possibilité d'être en contact avec une rivière poissonneuse et pas tellement (ou plutôt pas principalement) de pêcher des poissons.

27. J'étais sur le Lot hier, il est boueux mais péchable.

28. La Don est redevenuepêchable après le spate monstrueux du début de la semaine.

29. Ce soir en rentrant du travail, je m'arrête pour vérifier si l'Arve est pêchable.

30. Il faut que j'aille vers où pour que ce soit pêchable à la palme.

31. Un réservoir de pêche à la mouche à la truite de 15ha, péchable du bord.

Remarquons qu'assez souvent, l'adjectif est accompagné d'un adverbe (difficilement, tout à fait, encore, plus, toute la semaine) qui caractérise le degré ou le moment de «péchabilité »; dans tous les cas, c'est alors la rivière qui est caractérisée.

32. C'est très pêchable et très agréable.

33. Les postes situés à l'extérieur du Golfe étaient donc difficilement pêchables.

34. Une zone riche et variée pratiquement pêchable à la palme par tous les temps.

35. 17h la mer est en train de tomber un peu,et devient alors plus pêchable

36. Donc malgré des niveaux encore hauts, le Rio Grande fut quand même pêchable toute la semaine. 
Cette gradation dans la «péchabilité » (on nous pardonnera le néologisme), toujours associée au cours d'eau, et non au poisson, nous semble à nouveau aller dans le sens d'un lien direct avec la rivière, qui est plus ou moins péchable, plus ou moins accessible. Cette variation dans le contact témoigne de la relation privilégiée, facile ou contrariée, en tout cas soumise à des variations, du pêcheur avec le lieu de son activité. Au point que c'est cette relation qui devient l'objet de sa passion.

4.Remarques complémentaires au phénomène de transitivation

Ce phénomène de transitivation, qui, à notre avis, ne serait possible que dans des situations bien particulières, appelle des études plus approfondies dont nous allons donner quelques balises.

\subsection{Tests syntaxiques}

Bien que notre préoccupation ne soit pas strictement syntaxique, on peut voir que les tests généralement utilisés pour repérer des structures transitives peuvent fonctionnerpour les constructions [pêcher det rivière] ('Il s'agit bien chaque fois d'un objet direct, se prêtant aux tests habituels de pronominalisation, de passivation...[...]'.(Serbat, $1998: 220)$. Il faut toutefois noter que les exemples sont assez rares.

Passivation

37. mais bien sûr, plus tu montes, moins il y aura eu de monde, même si larivièreest pêchée tous les jours depuis un mois.

Pronominalisation

38. la Loire je la pêche du bord, en barque je ne connais pas, mais je doute qu'il y ait une grosse différence.

4.2 D’autres verbes concernés ou non

Verbes du domaine de la pêche 
Nous nous sommes demandé tout d'abord si d'autres verbes liés à l'activité de la pêche et pouvant se trouver à la place de pêcher dans notre structure de base pouvaient accepter le même type de transitivation (par exemple attraper: [attraper $\operatorname{det} \mathrm{Y}$ (poisson) dans det $\mathrm{Z}$ (rivière) avec det W (appât ou méthode)]. Nous avons ainsi testé : hameçonner, tuer, attraper, appâter. Aucun de ces verbes n'apparaît dans la structure avec construction directe [verbe + det + rivière].

On peut faire l'hypothèse que si seul le verbe pêcher autorise la transitivation de rivière, c'est parce qu'il se trouve dans la position la plus saillante dans la taxonomie de ces verbes (en position de terme de base pour reprendre la terminologie de la psychologue cognitive E. Rosch (Rosch et al., 1976)) : tuer et attraper sont plus généraux et ne s'appliquent pas qu'à la pêche, hameçonner et appâter sont plus spécifiques et correspondent plutôt à des méronymes de pêcher.

Verbes d'autres domaines

En revanche, ce phénomène de transitivationdu complément de lieun'est pas rare dans d'autres domaines. A partir de verbes renvoyant à une activité plus ou moins ludique, nous avons recherché si des compléments de lieu, que l'on s'attendrait à trouver introduits par des prépositions commedans ou sur, pouvaient apparaître en position d'objet direct. Nous avons trouvé plusieurs exemples parfois assez étonnants :

39. Essayant de nager un fleuve, elle est descendue au fond, à une grotte (conte de fées).

40. Quand il décida de pagayer unerivière de sa source à la mer, il choisit la plus grande du monde(site sur le canoe Kayak).

41. Bonjour, à partir de quand peut on généralement skier le mont blanc?(forum sur le ski).

42. Le skimboard consiste à surfer une vague en se lançant de la plage (wikipedia).

43. Rouler un vélo électrique est pour les uns un élément d'un style de vie extrêmement agréable et sain (site Alouette, d'un spécialiste de vélo). 
44. on peut pédaler un vélo de qualité à vitesses multiples (site Velec d'un marchand de vélos).

Ce qui pose question, c'est que ces énoncés dans lesquels un complément prépositionnel de lieu se trouve en position d'objet, semblent s'éloigner de la structure prototypique des énoncés transitifs. Que ce soit chez (Hopper et al., 1980), chez (Lazard, 1994) ou (Givon, 1984), on retrouve l'idée que l'objet doit être 'non-intentionnel, non-agissant et affecté' (Givon, 1984) ou encore que 'l'objet individué est pleinement affecté' (Lazard, 1994 : 248) avec un 'changement clairement visible ' (Givon, 1984). Or, dans aucun de ces exemples, l'objet ne semble vraiment 'affecté' (' un référent est affecté par un procés si le procès fait qu'il subisse un changement' (Larjavaara, 2000: 203). Si la transitivation de ces complémentsde lieu est acceptable, c'est donc bien parceque le locuteur veut leur donner une saillance particulière. En revanche, il est plus difficile de repérer un 'changement' visible. Si dans le jardinier a tué le lapin (exemple proposé par Lazard), on voit bien la modification de l'état de l'objet, c'est nettement moins clair avec la rivière, dont on ne voit pas très bien en quoi le fait de la pêcher amène à un changement d'état sauf si on la considère comme un objet animé qui, du fait de la pêche, s'est trouvé assailli et trituré et donc modifié. On trouve d'ailleurs trace de l'idée d'un combat dont la rivière est le lieu mouvant et qui, forcément s'en trouve affecté dans :

45. J'aime beaucoup pêcher la rivière car elle m'apporte des sensations au niveau combativité des poissons énormes ;

Cette hypothèse semble nettement moins défendable pour les exemples (rares toutefois) concernant l'objet vélo par exemple (43 et 44).

En revanche, c'est peut-être pour cette raison que nous n'avons trouvé aucune occurrence de *chanter un théâtre

*danser une scène. 
*Boxer un ring.

Dans ces trois exemples, qui seraient les équivalents avec transitivation d'énoncés canoniques avec complément de lieu, aucun des lieux ne peut être considéré comme affecté, modifié par l'action.

Un seul exemple correspondant à la structure[verbe det défini $\mathrm{N}$ de lieu] a été trouvé

46. Beaucoup de gens sont encouragés à danser la salle de séjour de leurs maisons

Ce qui est plus étonnant, c'est que nous avons trouvé très peu d'exemples correspondant à la structure : [chasser det lieu] hormis quelques cas : 1 de chasser la lande, 1 de chasser la bruyère, 3 de chasser la forêt, 3 dechasser les bois :

47. A la fin de la battue on peut chasser les bois et prairies environnantes, à l'aide de son chien d'arrêt à la recherche de bécasse ou palombes

Dans ces cas d'impossibilité ou de difficulté à transitiver le complément de lieu, on peut penser que ce lieu n'est pas considéré comme l'objet affecté et donc que l'agent ne perçoit pas un lien direct avec lui. Mais bien sûr, cette hypothèse devrait faire l'objet d'une analyse plus approfondie.

4.3 Phénomène trans-langues

Ce phénomène de transitivation d'un complémentde lieu n'est pas réservé au français, on le trouve aussi, toujours dans le domaine de la pêche, en anglais, en espagnol ou en italien:

48. River fishing tips for those who are new and need to know how to fish a river

49. una aventura de 3 díasacabalgata, para pescar un río en que las truchas que habitanahí

50. Sono d'accordo con tequandodiciche è un privilegioavere e pescare un fiumecosì!!

Cet aspect translinguistique renforce l'hypothèse d'un phénomène cognitivo-affectif qui instaurerait un lien privilégié entre le pêcheur et la rivière, relation que les pêcheurs s'autorisent à formuler lorsqu'ils se trouvent entre eux ou lorsqu'ils veulent faire part de ce 
lien étroit à l'extérieur, quitte à transgresser la formulation généralement admise. Au fond, dans toutes ces situations où, dans le cadre d'un loisir ou d'un sport, des acteurs sont amenés à avoir une activité dans un lieu particulier, c'est le lien avec ce lieu qui peut devenir le plus excitant, presque plus que l'activité elle-même (le pêcheur et la rivière, l'alpiniste et la montagne, le surfeur et la vague).

\section{Conclusion}

Cet article s'est concentré sur l'étude du phénomène de transitivation d'un lieu dans certains énoncés, en lien avec une situation de communication particulière qui fait intervenir, d'une part, un domaine spécialisé lié aux loisirs ou au sport et, d'autre part, une intense implication du locuteur dans l'activité concernée. Dans cette situation discursive, le locuteur s'autorise à transitiver le complément de lieu pour en faire l'objet ; il devient alors objet syntaxique de la phrase et objet d'intérêt du sujet (souvent le locuteur lui-même). A partir des occurrences trouvées sur l'internet correspondant à la structure [pêcher (prep)det rivière], nous avons pu montrer que la structure [pêcher det rivière] était relativement fréquente (plus de $17 \%$ des occurrences) et surtout, que, lorsqu'elle était utilisée, c'était très majoritairement (à plus de 75\%) dans des sites relevant du domaine de la pêche et d'une expression très personnalisée (blog, site personnel...). Ce constat confirme donc notre hypothèse, en tout cas pour le domaine de la pêche. De nombreuses perspectives s'ouvrent avec cette première étude. Tout d'abord, nous devons nous intéresser à la dimension systématisable, d'un point de vue sémantique, de l'hypothèse. Il est ainsi étonnant que ce phénomène soit nettement moins présent dans le domaine de la chasse ; il se peut que ce soit dû à la nature des lieux concernés, ou bien à la nature de l'activité mais il s'agira alors de comprendre pourquoi cette structure est impossible (en tout cas non trouvée sur l'internet) pour le domaine de la danse par exemple. L'aspect translinguistique est aussi intéressant. En effet, ce phénomène de 
transitivation semble exister dans d'autres langues (en tout cas pour la pêche), ce qui pourrait laisser penser qu'il s'agit d'un phénomène cognitivo-syntaxique (si on nous autorise le néologisme), comme certains auteurs le pensent déjà de la position objet. Dans le même temps, cette transitivation est bien moins présente dans certaines langues, comme l'italien, ce qui est tout de même étonnant. Du point de vue sémantico-syntaxique, nous n'avons examiné que des cas où c'est le complément de lieu qui est concerné. Or, d'autres compléments peuvent eux-aussi être transitivés (jouer contre l'espagnol/jouer l'espagnol par exemple). Il serait nécessaire d'évaluer les raisons sémantiques de ces possibilités ou non-possibilités (complément de temps par exemple).

La prise en compte de la dimension « socio-pragmatique » de la construction des énoncés, que nous examinons via la notion de genre textuel, rejoint en partie les préoccupations des grammaires de construction ( Désagulier, 2011) et, sans doute plus encore la perspective phénoménologique de l'analyse sémantique (Lebas, 2007). Nous ne sommes spécialiste ni de la première ni de la seconde. Il s'avère toutefois que, en nous plaçant dans la perspective de la prise en compte de données réelles, incontournables lorsque on étudie des discours spécialisés dont nous ne pouvons pas prétendre être locuteurs, nous sommes amenée à examiner des phénomènes dont les analyses rejoignent des questionnements de linguistique fondamentale.

S'il en était encore besoin, cela prouve qu'une linguistique qui prend les corpus spécialisés comme objet ne relève pas seulement d'une linguistique appliquée, souvent entendue comme une linguistique de seconde zone, mais bien d'une approche qui peut mettre en évidence des fonctionnements dont la pertinence peut être ensuite évaluée pour la langue dite générale, en l'occurrence, la possibilité de transitiver des compléments circonstanciels dans des situations d'implication affective forte

Enfin, le fait que cette transitivation, qui correspond à un écart par rapport à la norme, apparaisse dans des situations de communication particulières relevant de domaines 
spécialisés est aussi interpelant pour la terminologie. Il sera intéressant ainsi d'étudier ce qu'il se passe dans des domaines ne relevant pas du sport ou des loisirs mais des domaines techniques ou scientifiques, dans lesquels des experts peuvent être très impliqués (aéronautique, nautisme, biologie...). Il y a fort à parier que ce même type de dérogation à la règle puisse apparaître.

Bahtia, V.-K.(1993).Analysing Genre - Language Use in Professional Settings.London: Longman.

Baudouin, J.-M. (2004). Genre de texte et activité : le cas de l'autobiographie. Les modèles du discours face au concept d'action. Cahiers de Linguistique Française, Université de Genève, 26: 391-411.

Beauvisage, T. (2001). Morphosyntaxe et genres textuels. Exploiter des données morphosyntaxiques pour l'étude statistique des genres textuels : application au roman policier. TAL(TraitementAutomatique des langues),42.2: 579-608.

Biber, D. (1988). Variation Across Speech and Writing. Cambridge University Press.

Condamines, A.(2002). Corpus Analysis and Conceptual Relation Patterns. Terminology, 8.1: 141-162.

Condamines, A. (2009). L'expression de la méronymie dans les petites annonces immobilières ; comparaison français, anglais, espagnol. JFLS (Journal of French LanguageStudies), 19: 3-23.

Depecker, L. (1994). Pour les jargons. Meta: journal des traducteurs / Meta: Translators' Journal, 39.4: 736-740.

Désagulier, G. (2011). Le programme sociopragmatique des grammaires de constructions, bilan et perspectives.Intellectica 56(2): 99-123.

Desclés, J.-P. (1998). Transitivité sémantique, transitivité syntaxique. Dans : A. Rousseau (dir.), La Transitivité. Villeneuve d'Asq: Presses Universitaire du Septentrion,pp. 161180.

Deulofeu, J. (2000). Type d'énoncés et « genre » : le cas des commentaires sportifs.Dans : M. Bilger(dir.), Corpus, méthodologies et applications linguistiques. Paris: Champion, pp. 271-295.

Dowty, D. (1991). Thematic proto-roles and Argument selection.Language 67.3: 547-619.

Dubois, J. (1969). Grammaire structurale du français : la phrase et les transformations. Paris: Larousse. 
Fradin, B. (2003). Nouvelles approches en morphologie, Paris : PUF.

François, J.(2003). La prédication verbale et les cadres prédicatifs. Bibliothèque de l'Information Grammaticale 54, Louvain: Peeters.

Givon, T. (1984).Syntax: A functional-typological introduction. Amsterdam/Philadelphia: John Benjamins.

Hathout, N., Plénat M. et Tanguy L. (2003). Enquête sur les dérivés en -able. Cahiers de grammaire,28: 49-90.

Hopper, P-J. et Thompson, S.-A. (1980). Transitivity in Grammar and Discourse. Language, 56.2: 251-299.

Jørgensen, M.-W. et Phillips, L. (2002). Discourse Analysis as Theory and Method, London, Thousand Oaks, New Delhi: SAGE Publications Ltd.

Karlgren, J.etCutting D. (1994). Recognizing Text Genres with Simple Metrics Using Discriminant.Analysis.Proceedings of COLING'94, vol. 2, Kyoto, Japan: 1071-1075.

Lakoff, G.(1977). Linguistic Gestalts.Chicago Linguistic Society, 10: 236-287.

Larjavaara, M. (2000). Présence ou absence de l'objet. Limites du possible en français contemporain. Thèse de l'Université d'Helsinki.

Larjavaara, M. (2008). Penser (à) l'emploi : schémas actanciels dans deux genres de textes Version provisoire pour les pré-actes (mai 2008). www.helsinki.fi/nykykielet/congres/RSL/RSLpreactes/Larjavaara.rtf

Lazard, G. (1994). L'actance. Paris : PUF.

Lazard, G. (1998). De la transitivité restreinte à la transitivité généralisée. Dans : A. Rousseau (dir.) : La Transitivité. Villeneuve d'Asq : Presses Universitaire du Septentrion,pp. 5584.

Lebas, F. (2007). Pour une vision non dualiste de la transitivité. Dans : D. Bouchard, I. Evrard et E. Vocaj (dir.) Représentation du sens linguistique. Bruxelles: Duculot, De Boeck, pp. 151-162.

Leeman, D. (1992). Deux classes d'adjectifs en -ble.Langue française, 96 :44-64.

Marshman, E., M.-C. L'Homme etSurtees, V. (2008). Portability of cause-effect relation markers across specialized domains and text genres: A comparative evaluation. Corpora 3.2): 141-172.

Rousseau, A.(1998). La double transitivité existe-t-elle ?.Dans : A. Rousseau (dir.) : La Transitivité. Villeneuve d'Asq : Presses Universitaire du Septentrion, 55 : 85-112. 
Rogers, M. (2000). Genre and Terminology. Dans: A.Trosborg, (dir.),Analysing Professional Genres. Amsterdam/Philadelphia: John Benjamins,pp. 3-21.

Rosch, E., Merbvis, C. B., Gray, W. D., Johnson, D. M. etBoyes-Braem, P. (1976). Basic objects in natural categories .Cognitive Psychology, 8: 382-439.

Serbat, G. (1994). Le golf : une démocratisation et une transitivation galopantes. L'information grammaticale,62: 57-58.

Spang-Hanssen, E. (1973). Les prépositions incolores du français moderne. Université du Michigan, Gad.

Serbat, G. (1998). Préfixe et transitivation en latin. Dans: A. Rousseau (dir.) : La transitivité. Villeneuve d'Asq : Presses Universitaire du Septentrion, 55,pp. 219-232.

Wüster, E. (1981). L'étude scientifique générale de la terminologie, zone frontalière entre la linguistique, la logique, l'ontologie,l'informatique et les sciences des choses.Dans:G. Rondeau et H. Felber(dir.) :Textes choisis de terminologie. Québec : GIRSTERM, Université de Laval, pp. 55-108.

\begin{tabular}{|c|c|c|}
\hline $\begin{array}{c}\text { Construction [pêche à det } \\
\text { poisson] }\end{array}$ & $\begin{array}{c}\text { Construction [pêche de det } \\
\text { poisson] }\end{array}$ & Total \\
\hline $14914(56,83 \%)$ & $11327(43,17 \%)$ & 26241 \\
\hline
\end{tabular}

Tableau 1 : proportion de construction avec à vs de dans la structure comportant pêche

\begin{tabular}{|l|l|l|l|l|l|}
\hline & $\begin{array}{l}\text { Pêcher dans } \\
\text { det rivière }\end{array}$ & $\begin{array}{l}\text { Pêcher en } \\
\text { rivière }\end{array}$ & $\begin{array}{l}\text { Pêcher sur } \\
\text { (det) rivière }\end{array}$ & $\begin{array}{l}\text { Pêcher det } \\
\text { rivière }\end{array}$ & Total \\
\hline Forum & 78 & 113 & 24 & $95(30,5 \%)$ & 310 \\
\hline Blog & 110 & 44 & 23 & $89(33,5 \%)$ & 266 \\
\hline Récit & 88 & 13 & 28 & $35(21,5 \%)$ & 164 \\
\hline Presse & 26 & 11 & 0 & $8(18 \%)$ & 45 \\
\hline Livre & 49 & 44 & 5 & $7(6,5 \%)$ & 105 \\
\hline Voyages & 32 & 9 & 21 & $20(24 \%)$ & 82 \\
\hline Légal & 79 & 18 & 8 & $1(1 \%)$ & 106 \\
\hline Traduction & 23 & 1 & 0 & $0(0 \%)$ & 24 \\
\hline Dictionnaire & 11 & 0 & 0 & $4(26,5 \%)$ & 15 \\
\hline Divers & 177 & 152 & 26 & $2(0,5 \%)$ & 357 \\
\hline Total & $673(45,7 \%)$ & $405(27,8 \%)$ & $135(9,16 \%)$ & $261(17,7 \%)$ & 1474 \\
\hline
\end{tabular}

Tableau 2 : répartition selon le genre des quatre structures étudiées comportant pêcher et rivière(s)

\begin{tabular}{|c|l|l|l|l|l|}
\hline & $\begin{array}{l}\text { Pêcher dans } \\
\text { det rivière }\end{array}$ & $\begin{array}{l}\text { Pêcher en } \\
\text { rivière }\end{array}$ & $\begin{array}{l}\text { Pêcher sur } \\
\text { (det) rivière }\end{array}$ & $\begin{array}{l}\text { Pêcher det } \\
\text { rivière }\end{array}$ & Total \\
\hline $\begin{array}{c}\text { Site perso et } \\
\text { domaine pêche } \\
\text { par rapport }\end{array}$ & $186 / 673$ & $112 / 405$ & $21 / 135$ & $197 / 261$ & $516 / 1474$ \\
$(27,5 \%)$ & $(27,5 \%)$ & $(15,6 \%)$ & $(\mathbf{7 5 , 5 \% )}$ & $(35 \%)$ \\
aux autres sites & & & & & \\
\hline
\end{tabular}

Tableau 3 : répartition des quatre structures contenant pêche et rivière(s)en tenant compte de 


\begin{tabular}{|l|l|l|l|}
\hline $\begin{array}{l}\text { Pêcher dans } \\
\text { det rivière }\end{array}$ & $\begin{array}{l}\text { Pêcher en } \\
\text { rivière }\end{array}$ & $\begin{array}{l}\text { Pêcher sur } \\
\text { (det) rivière }\end{array}$ & $\begin{array}{l}\text { Pêcher det } \\
\text { rivière }\end{array}$ \\
\hline $186 / 516$ & $112 / 516$ & $21 / 516$ & $\mathbf{1 9 7 / 5 1 6}$ \\
$36 \%$ & $21,7 \%$ & $4 \%$ & $\mathbf{3 8 , 2 \%}$ \\
\hline
\end{tabular}

Tableau 4 : répartition des quatre structures dans les seuls sites relevant de la pêche et personnalisés

\begin{tabular}{|l|r|r|}
\multicolumn{2}{|c}{ pêche au } & pêche du \\
\hline Bar & 539 & 41 \\
\hline Brochet & 432 & 445 \\
\hline Thon & 517 & 425 \\
\hline Gros & 919 & 144 \\
\hline Carrelet & 477 & 28 \\
\hline Silure & 384 & 464 \\
\hline Loup & 178 & 279 \\
\hline Saumon & 619 & 447 \\
\hline Sandre & 301 & 405 \\
\hline Maquereau & 457 & 272 \\
\hline Homard & 456 & 214 \\
\hline Cormoran & 355 & 34 \\
\hline Plancton & 108 & 27 \\
\hline Poulpe & 376 & 156 \\
\hline Mulet & 187 & 189 \\
\hline Poisson & 463 & 461 \\
(+adj) & & \\
\hline Calamar & 144 & 59 \\
\hline Merlu & 136 & 93 \\
\hline Carnassier & 294 & 416 \\
\hline Couteau & 96 & 21 \\
\hline Flétan & 199 & 104 \\
\hline Black bass & 155 & 515 \\
\hline Marlin & 280 & 117 \\
\hline maigre & 108 & 114 \\
\hline Corail & 452 & 387 \\
\hline Bulot & 57 & 37 \\
\hline Mérou & 157 & 87 \\
\hline Barracuda & 90 & 84 \\
\hline Sailfish & 22 & 60 \\
\hline Bonefish & & 92 \\
\hline Gardon & 163 & 232 \\
\hline Tassergal & 26 & 46 \\
\hline
\end{tabular}




\begin{tabular}{|l|r|r|} 
Requin & 458 & 275 \\
\hline Corégone & 151 & 97 \\
\hline Cabillaud & 261 & 203 \\
\hline Hareng & 427 & 342 \\
\hline Krill & 68 & 68 \\
\hline Sambaza & 2 & 49 \\
\hline Crapet & 41 & 4 \\
\hline Congre & 162 & 152 \\
\hline Total & 10791 & 7685 \\
\hline
\end{tabular}

Tableau 5 : Noms de poissons ou de crustacés masculins, trouvés dans la position $\mathrm{N}$ dans la structure [pêche au/du N]

\begin{tabular}{|l|l|l|}
\hline & Pêche à la & Pêche de la \\
\hline Truite & 520 & 420 \\
\hline Moule & 195 & 25 \\
\hline Crevette & 455 & 301 \\
\hline Baleine & 480 & 430 \\
\hline Carpe & 470 & 455 \\
\hline Perche & 189 & 297 \\
\hline Daurade & 246 & 202 \\
\hline Seiche & 144 & 108 \\
\hline Sardine & 480 & 460 \\
\hline Tanche & 35 & 102 \\
\hline Coquille & 403 & 312 \\
\hline Morue & 480 & 451 \\
\hline Carangue & 26 & 79 \\
\hline Total & $4123(53 \%)$ & 3642 \\
\hline
\end{tabular}

Tableau 6 :Noms de poissons ou de crustacésféminins trouvés dans la position $\mathrm{N}$ dans la structure [pêche à/de la N]

\begin{tabular}{|l|l|}
\hline \multicolumn{1}{|l|}{ Coup } & \multicolumn{1}{l}{ Mouche } \\
\cline { 1 - 2 } Leurre & Traîne \\
\hline Quiver-typ & Ligne \\
\hline Tarpon & Torche \\
\hline Toc & Bombâts naturel \\
\hline Jigging & Palangre \\
\hline Surfcasting & Mitraillette \\
\hline Pain & Cuiller \\
\hline Chalut & Palangrotte \\
\hline Lancer & Teigne \\
\hline Posé & Tirette \\
\hline Filet & Lanterne \\
\hline Réservoir & Voile \\
\hline Feeder & Pelote \\
\hline JIG & Perle \\
\hline
\end{tabular}




\begin{tabular}{|l|l|}
\hline mouche & Plombée \\
\hline harpon & L'anglaise \\
\hline Spinnerbait & La bolognaise \\
\hline Bouchon & Roche \\
\hline Lamparo & \\
\cline { 1 - 1 } Fouet & \\
\cline { 1 - 1 } Krill & \\
\cline { 1 - 1 } grain &
\end{tabular}

Tableau 7 : noms de méthodes ou d'appâts trouvés en position $\mathrm{N}$ dans la structure [pêche à (le/la/les) N] 